\title{
Both Chargaff Second Parity Rule and the Strand Symmetry Rule Are Imprecise
}

\author{
Zhiyu Chen \\ Peiyou Education School, Shanghai, China
}

Email address:

Chenzhiyu@stu.cpu.edu.cn

\section{To cite this article:}

Zhiyu Chen. Both Chargaff Second Parity Rule and the Strand Symmetry Rule Are Imprecise. American Journal of Life Sciences. Vol. 6, No. 1, 2018, pp. 1-6. doi: 10.11648/j.ajls.20180601.11

Received: December 14, 2017; Accepted: March 5, 2018; Published: March 9, 2018

\begin{abstract}
In order to check Chargaff Second Parity Rule, we find the strands are asymmetric in human DNA, this breaks the strand symmetry rule. We calculate the ratio between oligonucleotide ATGC and oligonucleotide CGTA, and we compare the sample sequence average ratio ATGC/CGTA and the complementary sequence average ratio ATGC/CGTA. we find evolution degree more bigger, then the strand symmetry deviation will be more bigger. sequence and its complementary strand sequence obviously have two different characters, include physical property, chemical property and biological property. It is very important, base on this asymmetry, we can find some new and special theories in biology to explain how chromosome communicates and works in the future. we also find, both leukemia and breast cancer are weakening the DNA's asymmetry degree. Here need more research and check, maybe we can find an easy diagnosing method to leukemia and breast cancer, if this result here is right at last, it will benefit to the world.
\end{abstract}

Keywords: ATGC/CGTA, Strand Asymmetry, Complementary Sequence, Evolutionary Forces

\section{Introduction}

Strand symmetry

Chargaff Second Parity Rule holds that both\%A $\sim \% \mathrm{~T}$ and $\% \mathrm{G} \sim \% \mathrm{C}$ are valid for each of the two DNA strands [7]. This describes only a global feature of the base composition in a single DNA strand [1]. It is discovered by Austrian born chemist Erwin Chargaff [2] [6]. Hence, this double-stranded DNA characteristic can also be called "symmetry of complementary DNA strands [3] [4]". Chargaff's second parity rule ordinarily considers only mononucleotides, which have been extensively studied. The strand symmetry rule also says that oligonucleotide such as ATGC and its complementary oligonucleotide GCAT, there is the same relation $\mathrm{ATGC} \% \sim \mathrm{GCAT} \%$.

In figure 1, we can get the relation $\mathrm{ATGC} \%$ (strand $\mathrm{A}$ ) $\mathrm{ATGC} \%$ (strand $\mathrm{B}$ ), by the same way, we can get the relation about another oligonucleotide CGTA, between sample sequence and its complementary sequence, there are the same relation CGTA\%(strand A) CGTA\% (strand B), so there must be the relation, in strand $\mathrm{A}$, the ratio ATGC/CGTA approximately equals to the ratio ATGC/CGTA in strand B. This result is based on strand symmetry rule, it means two strands in one chromosome have approximate properties, they are symmetry. But it is not the fact, when we check the sequence using human DNA, and we find evolution degree more bigger, then the deviation will be more bigger, although many scientists have found this phenomenon for many years, they always look for some method to modify the big deviation, they try to make the strand symmetry rule to perfect all the species. We think to human, the stand is not symmetric, it is asymmetric. 


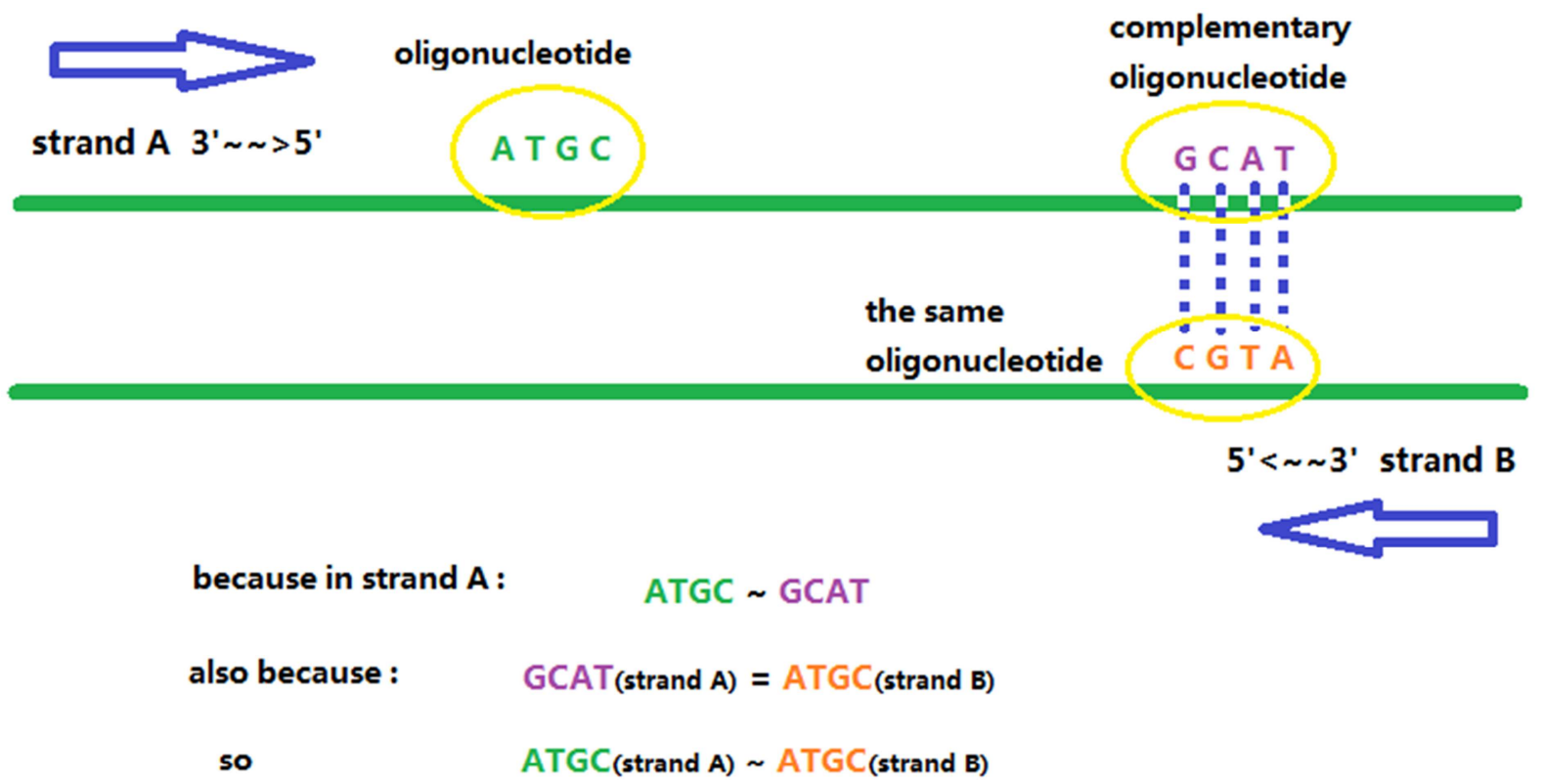

\section{That is to say : strand $A$ and strand $B$ are symmetric}

Figure 1. Analyze method to strand symmetry.

\section{Method}

See figure 2, segment a long sequence into many short segments with the same length, then, to every segment, calculate the number of oligonucleotide ATGC and the number of oligonucleotide CGTA. Then calculate the ratio ATGC/CGTA value for every segment. and give the average ratio value for the whole sequence

By $\mathrm{A} \sim \mathrm{T}, \mathrm{G} \sim \mathrm{C}$, translate out the complementary strand sequence code, use method above in figure 2, calculate the ratio ATGC/CGTA value for every segment. and give the average ratio value for the complementary strand [9] [10] sequence

Plot the ratio for the sequence and its complementary strand sequence

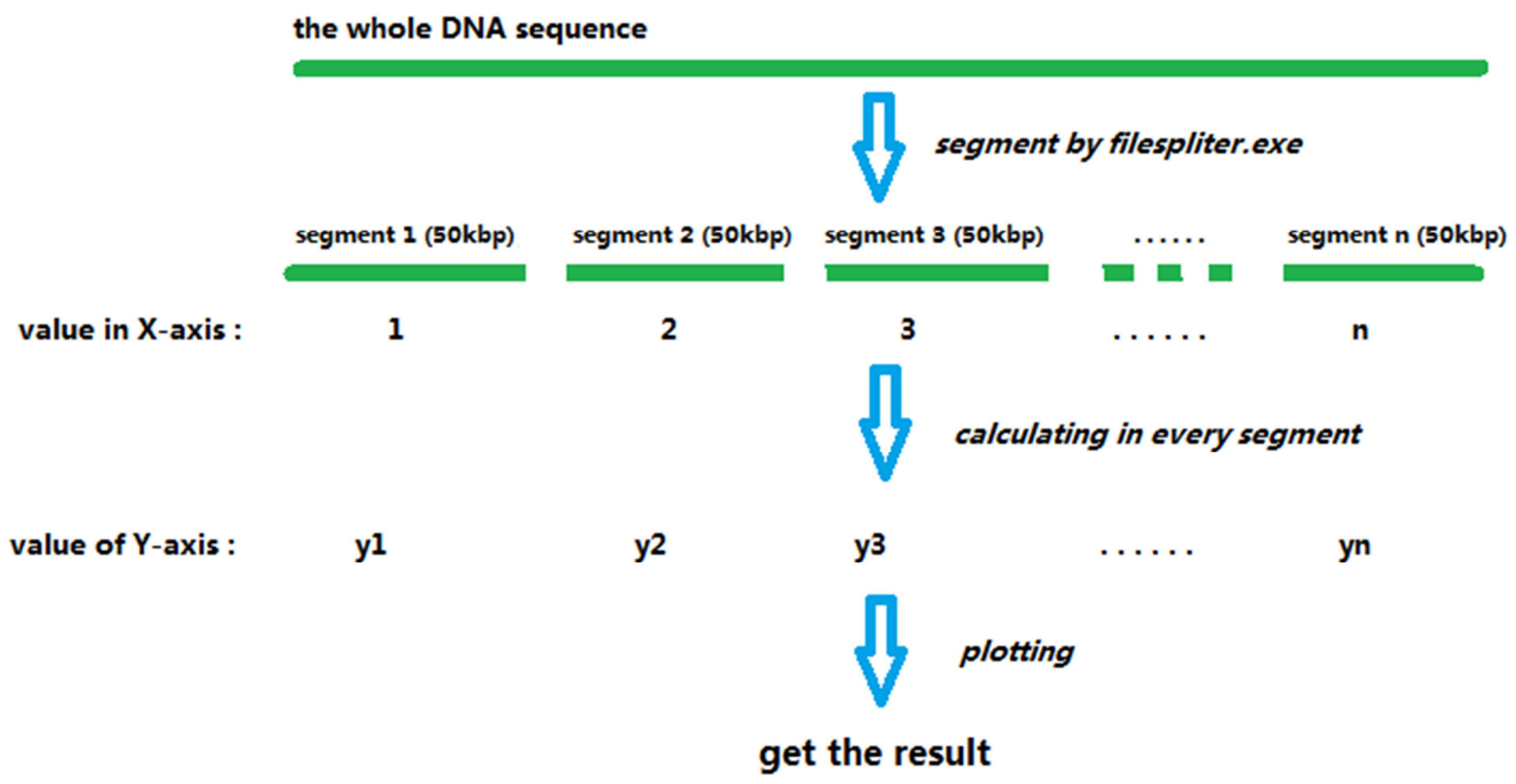

Figure 2. Calculate method.

\section{Results}

We use human DNA [8] sequence and mouse DNA sequence and fly DNA sequence to check strand symmetry, compare two strands' ATGC/CGTA, we compare the sample sequence's ATGC/CGTA value with its complementary strand sequence's ATGC/CGTA value.

Here is human's DNA sequence is hg19 sequence 


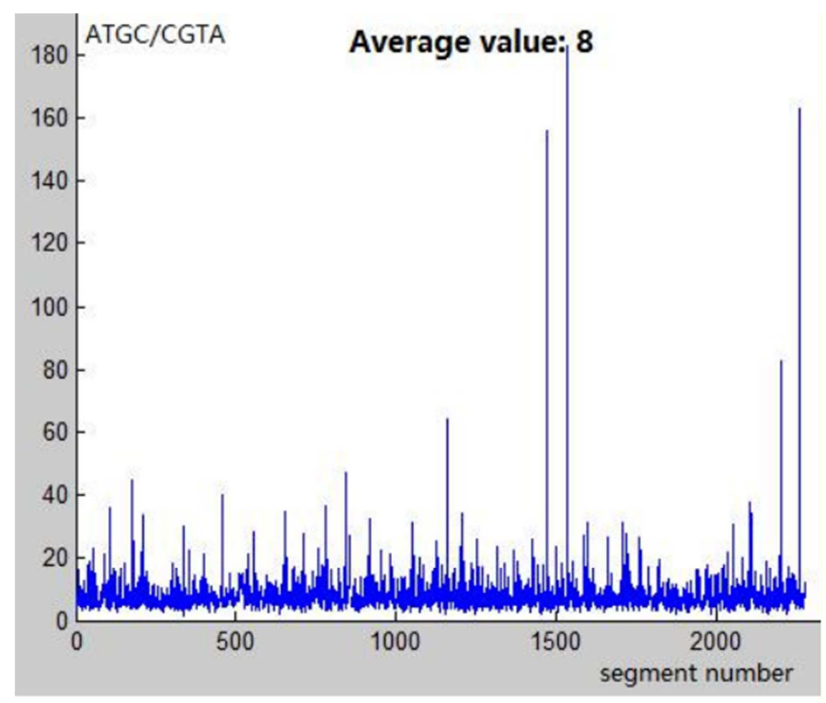

ATGC/CGTA distribution in hi19 sequence

Figure 3. Human healthy DNA.

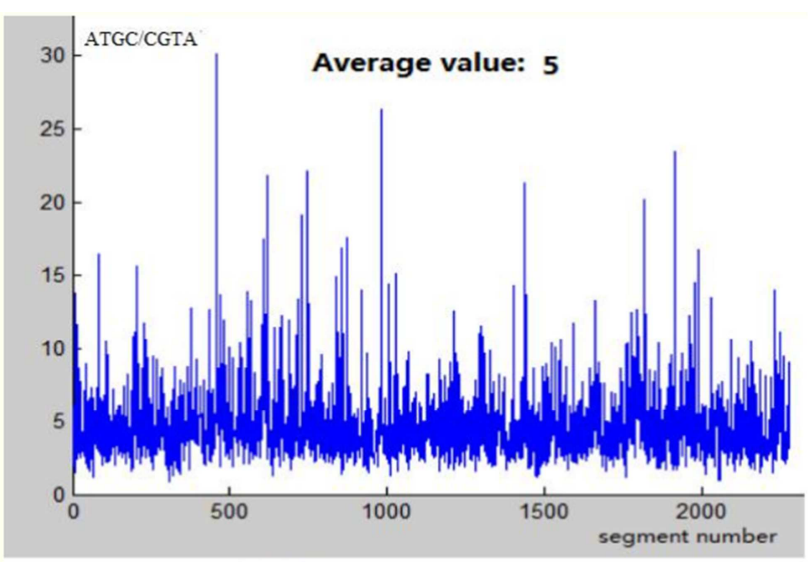

ATGC/CGTA distribution in hg19 complementary sequence

Figure 4. Human healthy DNA complementary sequence.

Then, we do the same analysis on a mouse's DNA, the sequence is from www.ncbi.nlm.nih.gov, the GenBank: NC_008802.1

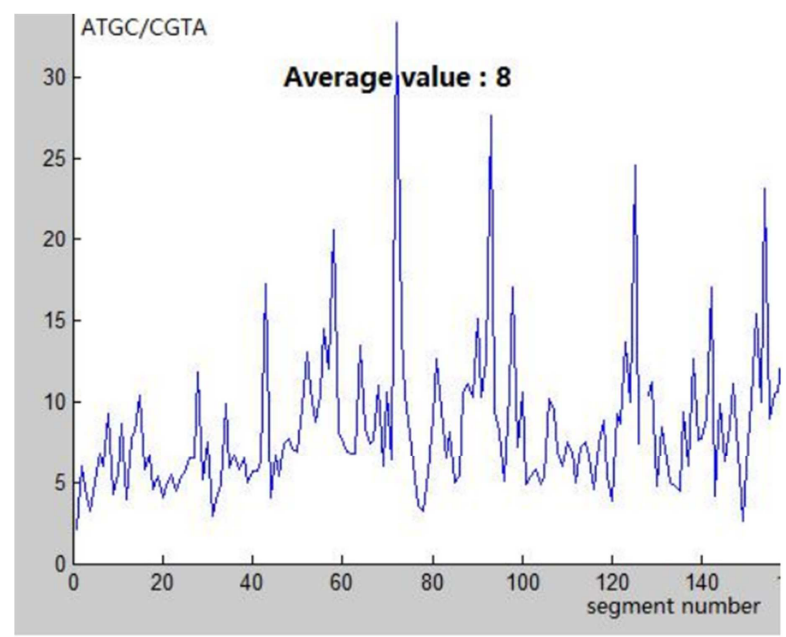

ATGC/CGTA distribution in mouse DNA sequence

Figure 5. Mouse DNA.

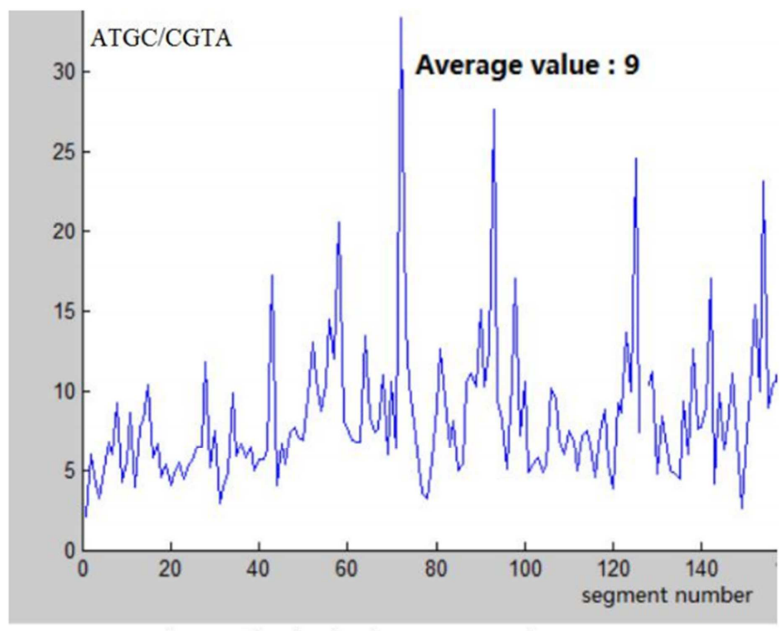

ATGC/CGTA distribution in mouse complementary sequence

Figure 6. Mouse DNA complementary sequence.

Next, we do the same analysis on a fly's DNA, the sequence is from www.ncbi.nlm.nih.gov, the GenBank: CP012523.1

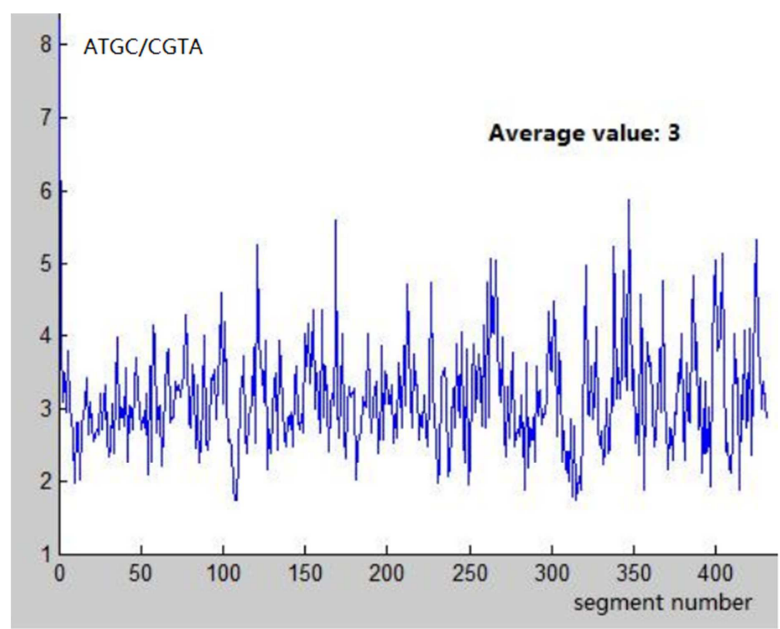

ATGC/CGTA distribution in fly DNA sequence

Figure 7. Fly DNA.

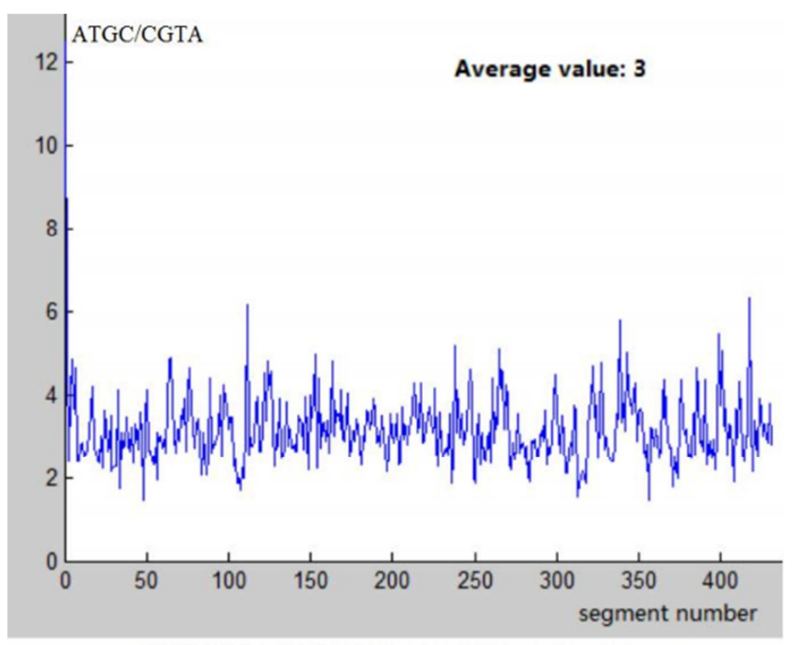

ATGC/CGTA distribution in fly complementary sequence

Figure 8. Fly DNA complementary sequence. 
Compare the above data using the following

Table 1. Asymmetry degree and Evolution degree.

\begin{tabular}{llllll}
\hline Species & Sequence ATGC/CGTA & Complementary sequence ATGC/CGTA & Ratio (big/small) & Asymmetry degree & Evolution degree \\
\hline fly & 3 & 3 & 1.0 & low & low \\
mouse & 9 & 8 & 1.1 & middle & middle \\
human & 8 & 5 & 1.6 & high & high \\
\hline
\end{tabular}

\section{Discussion}

First, we can find, both Chargaff Second Parity Rule and the Strand Symmetry Rule do not perfect to human DNA, in human chromosome, sequence and its complementary strand sequence obviously have two different characters, include physical property, chemical property and biological property. It is very important, based on this asymmetry, we can find some new and special theories in biology to explain how chromosome communicates and works in the future.

Here we also give the answer to question:"Did evolutionary forces select for the Chargaff ratios in single DNA strands, with equality of complementary oligonucleotide frequencies being an automatic consequence? Alternatively, did evolutionary forces select for equality of complementary oligonucleotide [5] frequencies, with the Chargaff ratios being an automatic consequence?" Evolutionary forces select for the Chargaff ratios in single DNA strands.

\section{More Application}

Next we do the same analysis on human breast cancer's DNA, the sequence is from www.ncbi.nlm.nih.gov

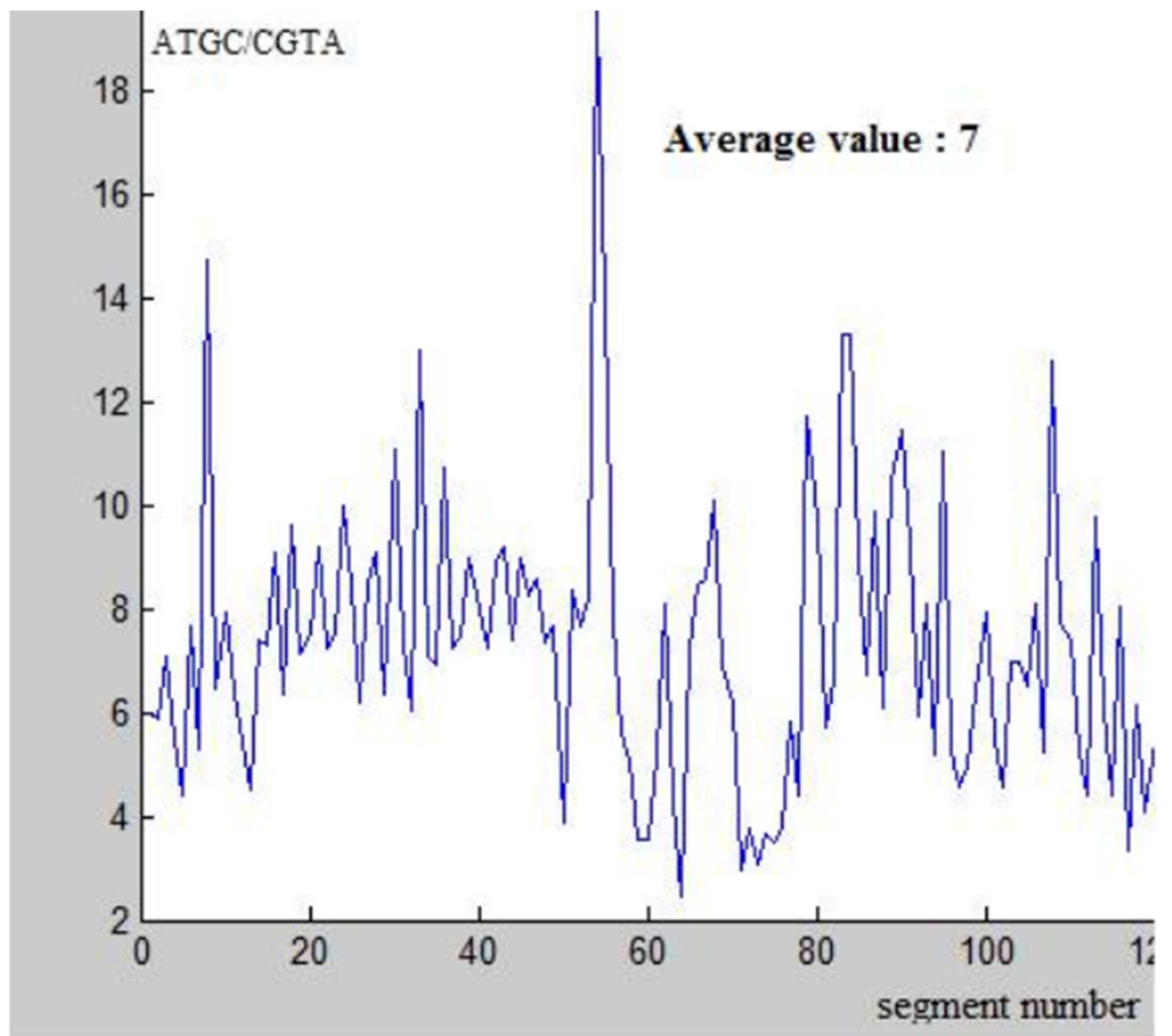

ATGC/CGTA distribution in human breast cancer DNA

Figure 9. Human breast cancer DNA. 


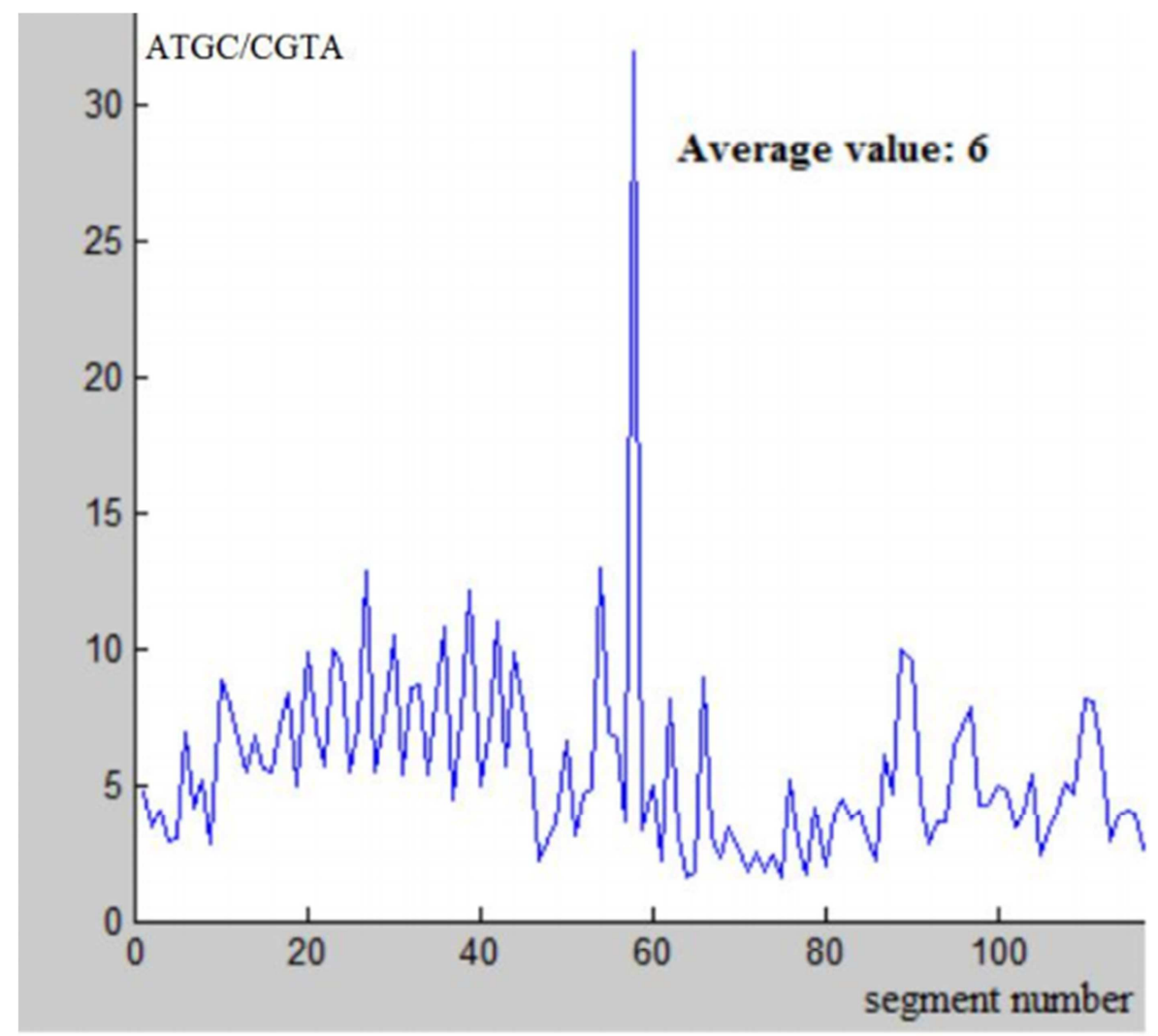

ATGC/CGTA distribution in human breast cancer complementary sequence

Figure 10. Human breast cancer DNA complementary sequence.

Next we do the same analysis on human leukemia's DNA, the sequence is from www.ncbi.nlm.nih.gov

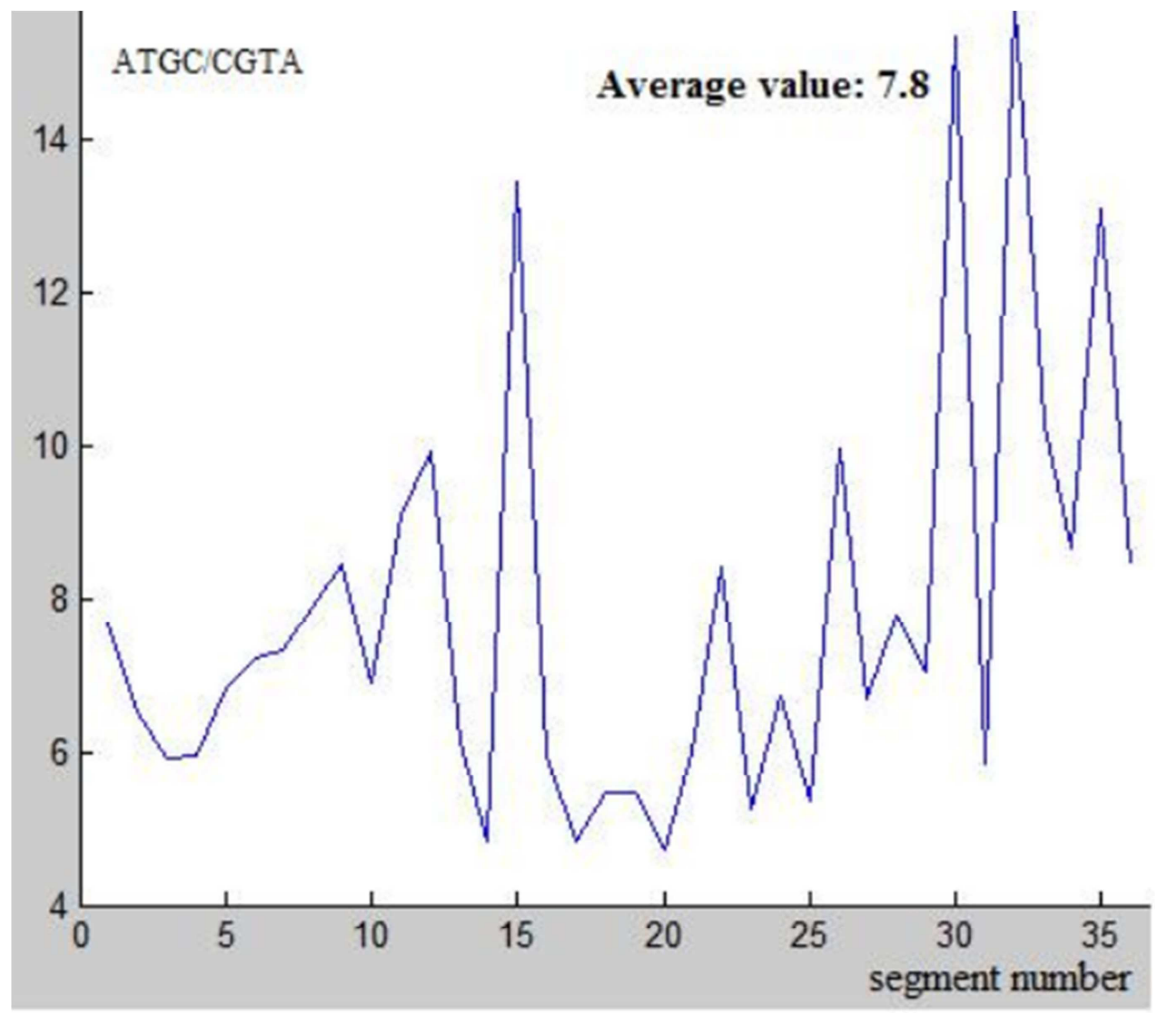

ATGC/CGTA distribution in leukemia DNA

Figure 11. Human leukemia DNA. 


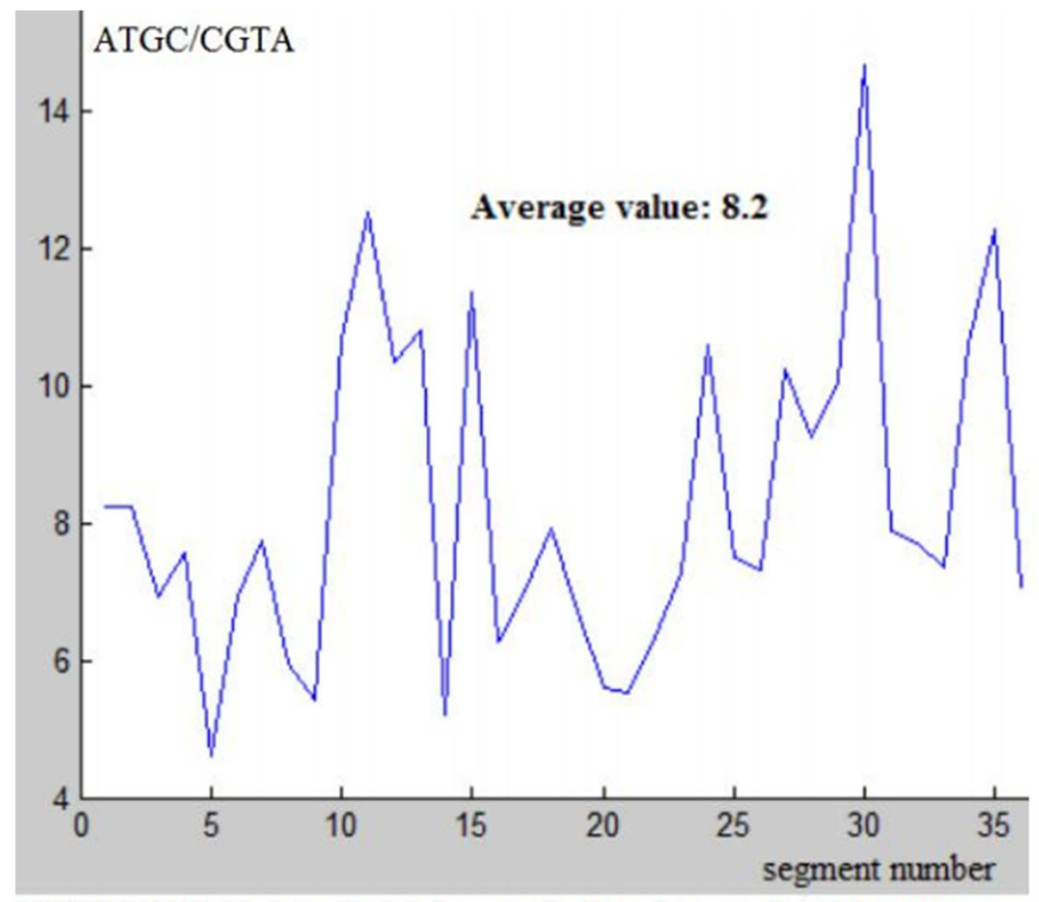

ATGC/CGTA distribution in human leukemia complementary sequence

Figure 12. Human leukemia DNA complementary sequence.

Compare the above data using the following

Table 2. Cancer and Asymmetry degree.

\begin{tabular}{lllll}
\hline Species & Sequence ATGC/CGTA & Complementary sequence ATGC/CGTA & Ratio (big/small) & Asymmetry degree \\
\hline Human healthy DNA & 8 & 5 & 1.6 & high \\
Homo Leukemia & 8.2 & 7.8 & 1.05 & 1.17 \\
Homo breast cancer & 7 & 6 & lower & low \\
\hline
\end{tabular}

Both leukemia and breast cancer are weakening the DNA's asymmetry degree. Here need more research and check, maybe we can find an easy diagnosing method to leukemia and breast cancer, if my result here is right at last, it will benefit to the world, thanks to other researchers!

\section{References}

[1] Rudner, R; Karkas, JD; Chargaff, E (1967). "Separation of B. Subtilis DNA into complementary strands. 3. Direct analysis". Proceedings of the National Academy of Sciences of the United States of America. 60 (3).

[2] Chargaff E, Lipshitz R, Green C (1952). "Composition of the deoxypentose nucleic acids of four genera of sea-urchin". J Biol Chem. 195 (1): 155-160.

[3] Baisnée PF, Hampson S, Baldi P: Why are complementary DNA strands symmetric?. Bioinformatics. 2002.

[4] Prabhu VV: Symmetry observations in long nucleotide sequences. Nucleic Acids Res. 1993, 21: 2797-2900. 10.1093/nar/21.12.2797.

[5] Forsdyke DR. 1995a. Relative roles of primary sequence and $(\mathrm{G}+\mathrm{C}) \%$ in determining the hierarchy of frequencies of complementary trinucleotide pairs in DNAs of different species. J. Mol. Evol. 41:573-591.
[6] Elson D, Chargaff E (1952). "On the deoxyribonucleic acid content of sea urchin gametes". Experientia. 143-145. doi:10.1007/BF02170221. PMID 14945441.

[7] Rudner, R; Karkas, JD; Chargaff, E (1968). "Separation of B. Subtilis DNA into complementary strands. 3. Direct analysis". Proceedings of the National Academy of Sciences of the United States of America. 60 (3): 921-2. doi: 10.1073/pnas.60.3.921. PMC 225140? Freely accessible. PMID 4970114.

[8] Perez, J.-C. (September 2010). "Codon populations in singlestranded whole human genome DNA are fractal and finetuned by the Golden Ratio 1.618". Interdisciplinary Sciences: Computational Life Science. 2 (3): $228-240$. doi: 10.1007/s12539-010-0022-0.

[9] Pray, Leslie (2008). "Discovery of DNA structure and function: Watson and Crick". Nature Education. 1 (1): 100. Retrieved 27 November 2013.

[10] Kosaka, N; Yoshioka, Y; Hagiwara, K; Tominaga, N; Katsuda, T; Ochiya, T (Sep 5, 2013). "Trash or Treasure: extracellular microRNAs and cell-to-cell communication". Frontiers in Genetics. 4: 173. doi:10.3389/fgene.2013.00173. PMC 3763217 Freely accessible. PMID 24046777. 